\title{
COVID 19 E OS IMPACTOS NA EDUCAÇÃO: PERCEPÇÕES SOBRE BRASIL E CUBA
}

\section{COVID-19 AND ITS EDUCATIONAL IMPLICATIONS: INSIGHTS INTO BRAZIL AND CUBA}

\author{
Eva Teixeira dos Santos \\ Programa de Pós-Graduação em Geografia - PPGGEO/CPAQ \\ Universidade Federal de Mato Grosso do Sul - UFMS, Brasil \\ evasantos.ufms@gmail.com \\ Eros Salinas Chavez \\ Programa de Pós-Graduação em Geografia - PPGGEO/CPAQ \\ Universidade Federal de Mato Grosso do Sul - UFMS, Brasil \\ salinaschavezeros@gmail.com
}

Anderson Antonio Molina da Silva Programa de Pós-Graduação em Geografia, Campus de Aquidauana - UFMS molina.and.molina@gmail.com

Geovandir André Lordano Programa de Pós-Graduação em Geografia - PPGGEO/CPAQ Universidade Federal de Mato Grosso do Sul - UFMS, Brasil geovandirlordano@gmail.com
Lucy Ribeiro Ayach
Programa de Pós-Graduação em Geografia - PPGGEO/CPAQ Universidade Federal de Mato Grosso do Sul - UFMS, Brasil lucy.ayach@ufma.br

Vicentina Socorro da Anunciação Programa de Pós-Graduação em Geografia - PPGGEO/CPAQ Universidade Federal de Mato Grosso do Sul - UFMS, Brasil vique56@hotmail.com

Ricardo Lopes Batista

Programa de Pós-Graduação em Geografia - PPGGEO/CPAQ Universidade Federal de Mato Grosso do Sul - UFMS, Brasil ricardo.batista@ufms.br

\section{RESUMO}

A Pandemia da Covid 19 tem causado sobre a população mundial impactos diretos na saúde física e mental, economia, política e relações sociais, dentre elas a educação. Nesta perspectiva, este trabalho analisa os efeitos da pandemia na área da educação no contexto espacial do Brasil e de Cuba, identificando as medidas adotadas, uma vez que o processo de aprendizagem depende diretamente do bem-estar, da saúde física e mental de alunos, professores e familiares. A partir das discussões apresentadas, destaca-se a ausência por parte dos governos dos países de um levantamento estatístico sobre a realidade, sobre as desigualdades regionais, do ponto de vista das infraestruturas nos países e sobre o acesso a internet por professores e alunos no caso do Brasil, uma vez que Cuba por dificuldades de acesso a rede mundial de computadores optou por tele aulas. Com isso, a dinâmica das famílias com crianças e adolescentes tem exigido um esforço maior dos pais, responsáveis e/ou cuidadores, que também é um impacto a ser considerado, uma vez que grande parte não possui preparação para a educação a distância no lar. Portanto, as medidas até agora adotadas são questionáveis quanto aos aspectos qualitativos não considerados no processo ensino aprendizagem e necessitam de mais reflexões e debates em torno da modalidade de ensino proposta frente ao contexto da pandemia, principalmente na educação básica.

Palavras-Chave: Covid-19. Impactos Educacionais. Brasil. Cuba.

Recebido em: 08/05/2020

Aceito para publicação em: 21/05/2020 
Covid 19 e os impactos na educação: percepções sobre Brasil e Cuba
Eva Teixeira dos Santos Eros Salinas Chavez Anderson Antonio Molina da Silva

Geovandir André Lordano Lucy Ribeiro Ayach

Vicentina Socorro da Anunciação Ricardo Lopes Batista

\begin{abstract}
The Covid-19 pandemic has caused on the global population direct impact on physical and mental health, economy, politics, and social relations, like education. Hence, this work analyses the pandemic effects on education in the spatial context of Brazil and Cuba, identifying implemented measures since the learning process depends directly on the wellbeing, physical and mental health of students, teachers, and relatives. From the presented discussions stands out the absence from both countries governments of a statistical survey on reality, regional inequalities, the countries facilities and on internet access by teachers and students in Brazil, once Cuba due to internet connection issues has opted for tv-lessons. Thus, the families' dynamic with children and teenagers has demanded much effort from parents, guardians and/or caregivers, which is also an impact to be considered since most of them are not prepared to distance learning in their homes. Therefore, the implemented measures so far are questionable in terms of qualitative aspects not considered in the learning process and requiring reflections and debates around the proposed teaching method in the pandemic context, especially upon basic education.
\end{abstract}

Key-words: Covid-19. Educational Implications. Brazil. Cuba.

\title{
INTRODUÇÃO
}

É consenso entre os pesquisadores e a sociedade que a pandemia da Covid-19 causou em todos os países do mundo uma saturação e colapso sem precedentes nos serviços de saúde, que exigiram a implementação de estratégias, nem sempre bem organizadas, para atender às necessidades resultantes do aumento progressivo da demanda por atendimento médico. A resposta dos serviços de saúde começa pela comunidade, com a educação da população, medidas de saúde pública e de prevenção por meio da atenção primária, além da coordenação e articulação com os hospitais. A ampliação da capacidade de resposta implica reforçar a atenção primária e a disponibilidade de recursos suficientes no âmbito hospitalar, por meio de medidas de reconversão de leitos, realocação da equipe de saúde, alta precoce dos pacientes, entre outras medidas. À medida em que essas capacidades da rede de serviços de saúde precisam ser reforçadas, ou que tenham sido superadas, é necessário considerar outras estratégias no nível comunitário.

A Covid-19 surgiu em Wuhan, na China, no final de 2019, e as medidas adotadas de testagem ampla e isolamento social, surtiram efeito para evitar que o coronavírus chegasse nas maiores cidades chinesas. No entanto, apesar do esforço, chegou no país vizinho, a Coreia do Sul, e mais uma vez a estratégia de testagem ampla e isolamento social conseguiram conter o avanço do coronavírus. Parecia que tais medidas seriam suficientes para conter o avanço da epidemia, e que esta ficaria restrita ao sudeste asiático. Mas, em um mundo globalizado, com um grande fluxo de pessoas que cruzam fronteiras diariamente, é muito difícil impedir que um vírus, altamente transmissível, alcance todo o planeta, se tornando uma pandemia. Chegou à Europa, América do Norte, e o cenário mundial vai se tornando a cada dia mais trágico, pois novos casos se multiplicam na África e América do Sul. Fatores socioeconômicos são determinantes no processo saúde-doença e a estratégia de isolamento social, adotada como medida de redução da vulnerabilidade da população, esbarra em um quadro social complexo agravado pela crise econômica que atinge países em desenvolvimento, como o Brasil (FARIAS, 2020).

Atualmente, a preocupação com a saúde humana e sua relação com o ambiente são assuntos bastante discutidos. Nesse processo, especialmente a Geografia da Saúde exerce papel relevante, pois os aspectos sociais e ambientais são na maioria das vezes os grandes responsáveis pelos problemas que afligem a saúde da população. Tal preocupação não é recente, visto que desde a antiguidade já eram feitas conexões entre ambiente e enfermidade. E é nesse contexto que a Geografia exerce um papel crucial, pois o estudo da superfície terrestre, da paisagem e da relação entre o homem e a natureza torna-se essencial, uma vez que os fatores sociais, econômicos e ambientais são alguns dos fatores que mais favorecem a disseminação das doenças. Os processos e problemas da globalização, os 
Covid 19 e os impactos na educação: percepções sobre Brasil e Cuba
Eva Teixeira dos Santos Eros Salinas Chavez Anderson Antonio Molina da Silva

Geovandir André Lordano Lucy Ribeiro Ayach Vicentina Socorro da Anunciação Ricardo Lopes Batista

fatores endêmicos e epidêmicos relacionados à saúde, bem como o ressurgimento de "velhas novas" epidemias, abriu-se campo significativo para atuação.

Assim, considerando a chegada da pandemia em 2020 no Brasil, este trabalho pretende analisar os efeitos da pandemia na área da educação tendo como recorte espacial Brasil e Cuba com o objetivo de mensurar e analisar os efeitos positivos e negativos de medidas adotadas, uma vez que o processo de aprendizagem depende diretamente do bem-estar, da saúde física e mental de alunos, professores e familiares. Portanto, o apontamento de informações deve ser analisado com muito cuidado, considerando que no setor da saúde os efeitos negativos (ou a morte) ocorrem a curto prazo e no caso da educação, na maioria das vezes, os efeitos negativos (ou a morte) ocorrem a longo prazo e muitas de forma imperceptível. Entender como os diferentes lugares, que tem características econômicas, ambientais, sociais e culturais diversificadas, influenciam na saúde das pessoas se torna essencial na Geografia da Saúde, que pode ajudar nos problemas referentes à saúde, se tiver como eixo norteador o ambiente, a sociedade e o território.

\section{CONSIDERAÇÕES SOBRE A PANDEMIA E OS IMPACTOS NA EDUCAÇÃO}

A Pandemia da Covid-19 afetou, no contexto mundial, não somente a saúde da população, mas, conjuntamente, aspectos econômicos, sociais e políticos, a ciência humana e social, em especial a geográfica, que congrega tais estudos de forma integrada e que exerce um papel preponderante para reflexões, análises e proposições no entendimento dos respectivos fatores, que são indissociáveis.

Diante de uma situação totalmente nova, com muitas lacunas obscuras na ciência, é de consenso que a busca de contribuições para o enfrentamento da doença e de suas repercussões só proporcionará resultados efetivos se forem consideradas de forma integrada.

Em 24 de março de 2020, a UNESCO publicou em seu site as principais repercussões, sendo que muitas delas vão mais além do setor da educação, para ajudar os países a prever e mitigar os problemas. Entre esses efeitos, constam os seguintes: a interrupção da aprendizagem; a nutrição; proteção; os pais não estão preparados para a educação a distância no lar; acesso desigual aos portais de aprendizagem digital; lacunas no cuidado das crianças; altos custos econômicos; maior pressão sobre as escolas e os sistemas escolares que permaneciam abertos naquele momento; aumento das taxas de evasão escolar; e, isolamento social (UNESCO, 2020a).

Desta forma, para analisar os aspectos quanto à reação e planejamento das diferentes regiões do país deve ser considerado também essas peculiaridades inter-regionais que se diferem, bem como diferenciações intra-regionais. As figuras 01 e 02 abaixo demonstram a diferenciação de números de casos e óbitos por Unidades da Federação (Brasil) e Províncias (Cuba), registrados até o final de abril de 2020.

Figura 01 - Distribuição dos casos (A) e óbitos (B) por Covid-19 por Unidade da Federação. Brasil, 2020.
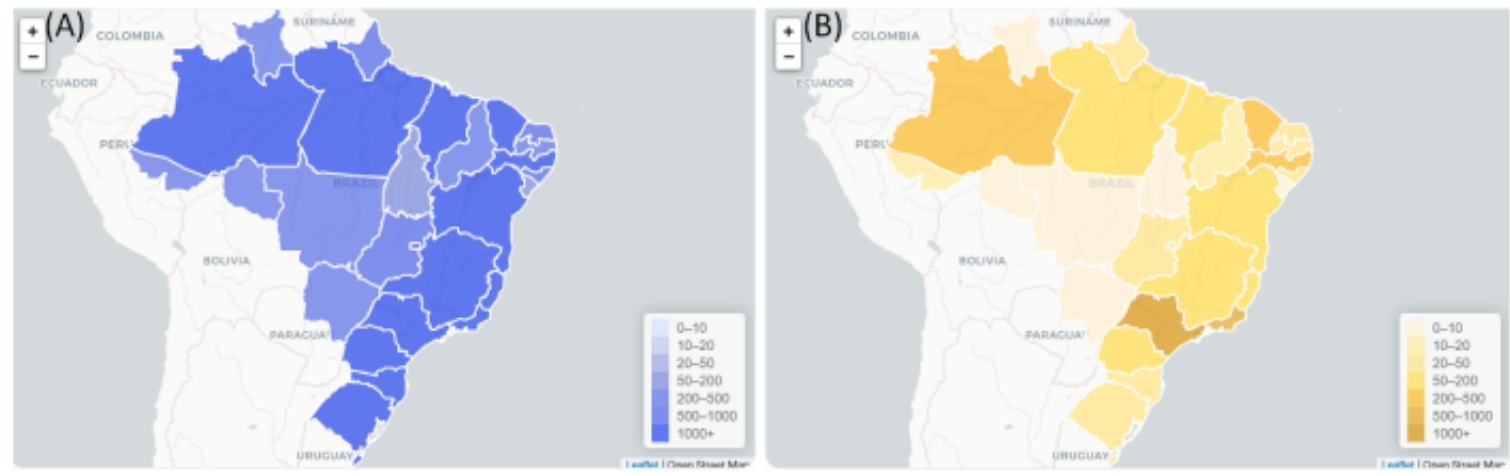

Fonte: https://covid.saude.gov.br/ - atualizado em 26/04/2020 às 16:30h. 
Covid 19 e os impactos na educação: percepções sobre Brasil e Cuba
Eva Teixeira dos Santos Eros Salinas Chavez Anderson Antonio Molina da Silva

Geovandir André Lordano Lucy Ribeiro Ayach Vicentina Socorro da Anunciação Ricardo Lopes Batista

Figura 02 - Distribuição dos casos confirmados e óbitos por Covid-19 por Províncias. Cuba, 2020.

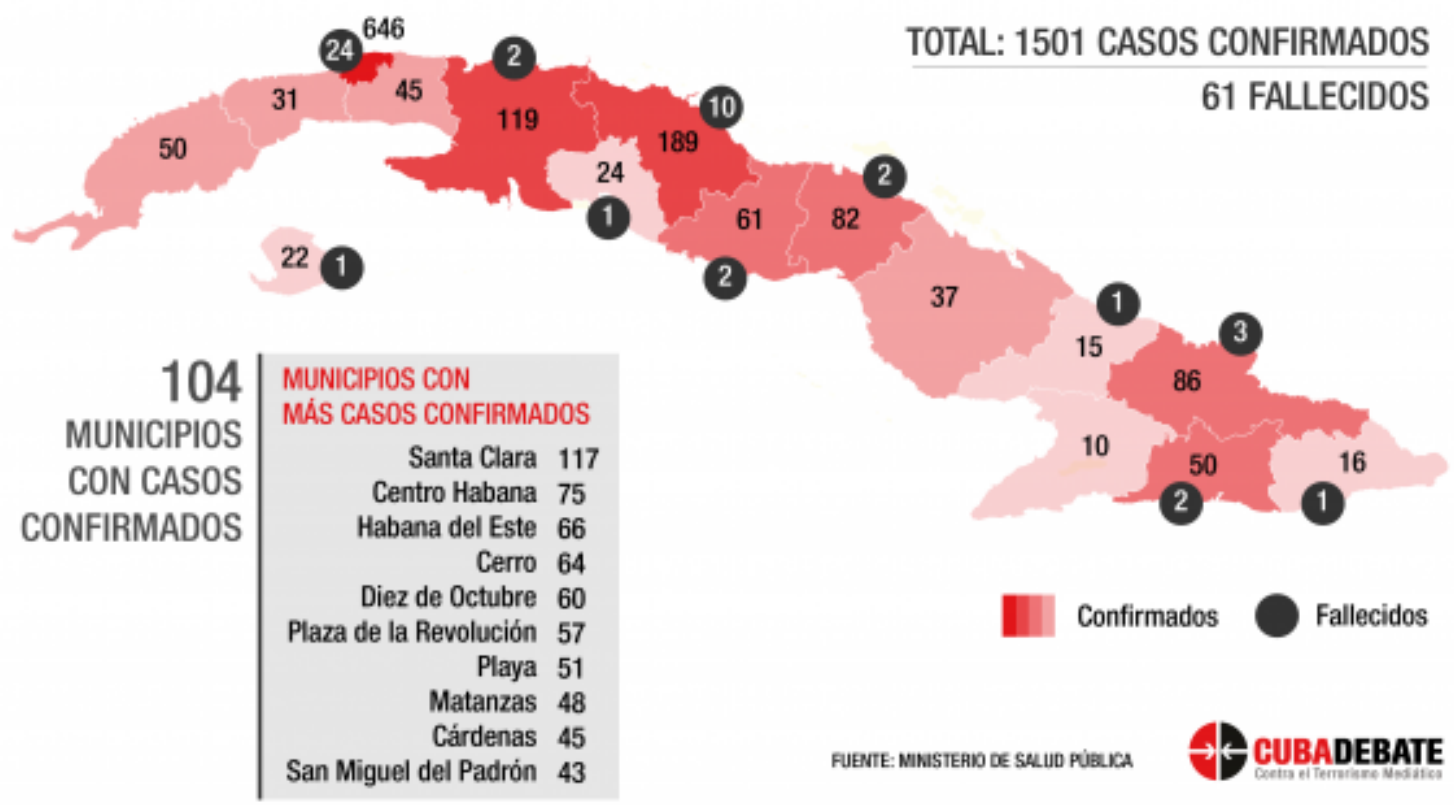

Fonte: CUBADEBATE.CU, 2020 (atualizado em 29/04/2020).

A partir desses dados é possível identificar várias implicações da Covid-19 sobre a sociedade brasileira e cubana. Uma implicação importante, no caso do Brasil, está relacionada quantidade de crianças que perderam os programas de alimentação escolar das quais dependem, outras foram expostas ou até sofreram violência dentro de casa, e muitas eram suscetíveis aos riscos aumentados de saúde sexual e reprodutiva que surgem quando as escolas fecham por mais do que algumas semanas. As rigorosas medidas de isolamento, o fechamento de escolas e locais de trabalho e a perda de renda para muitas famílias tiveram e continuarão a ter impactos negativos significativos sobre a educação, a saúde mental (causando estresse, preocupação e ansiedade) e o bem-estar das pessoas. Tais aspectos são evidenciados por Ornel let al. (2020, p. 3):

Como os custos econômicos associados aos transtornos mentais são altos, a melhoria das estratégias de tratamento em saúde mental pode levar a ganhos tanto na saúde física quanto no setor econômico. Além de um medo concreto da morte, a pandemia do COVID-19 tem implicações para outras esferas: organização familiar, fechamento de escolas, empresas e locais públicos, mudanças nas rotinas de trabalho, isolamento, levando a sentimentos de desamparo e abandono. Além disso, pode aumentar a insegurança devido às repercussões econômicas e sociais dessa tragédia em larga escala.

No que se refere a alimentação escolar, o alto número de crianças que sofrem a falta das refeições vitais na escola foi destacado por Carmen Burbano de Lara, Diretora da Divisão de Alimentação Escolar do Programa Mundial de Alimentação ao afirmar que "369 milhões de crianças estão perdendo refeições na escola em todo o mundo, com crianças em quase todos os países afetados", disse Burbano (UNESCO, 2020a).

Para minimizar esse problema, alguns países estão implementando práticas inovadoras para manter a saúde e o bem-estar no contexto da pandemia da Covid-19. Na Escócia, por exemplo, o governo criou 'hubs' com apoio de voluntários para prestar serviços às famílias, incluindo trabalhadores de serviços essenciais e o fornecimento de refeições escolares gratuitas, assistência infantil e acesso a assistências sociais. Na Jamaica, o governo está apoiando professores a propiciar aulas de educação 
Covid 19 e os impactos na educação: percepções sobre Brasil e Cuba
Eva Teixeira dos Santos Eros Salinas Chavez Anderson Antonio Molina da Silva

Geovandir André Lordano Lucy Ribeiro Ayach Vicentina Socorro da Anunciação Ricardo Lopes Batista

em saúde para alunos do ensino fundamental e médio via plataformas de aprendizado remoto, inclusive por meio de modalidades on-line e de transmissão, sobre temas que incluem alimentação e condicionamento físico adequados, bem-estar emocional e mental e saúde sexual e reprodutiva (UNESCO, 2020b).

\section{O IMPACTO DA PANDEMIA DE COVID-19 NA EDUCAÇÃO NO BRASIL E CUBA}

É fato que dentre todos os reflexos das ações de isolamento social por conta da pandemia de coronavírus a educação domiciliar ou homeschooling, passou a fazer parte da rotina de mais de um bilhão de alunos desde o final de fevereiro até o momento, conforme aponta o monitoramento da Unesco (Organização das Nações Unidas). De acordo com as informações disponíveis no site, no final de fevereiro 79.435.099 estudantes foram afetados com o fechamento total de escolas em dois países, sendo respectivamente China e Mongólia, conforme figura $03(\mathrm{~A})$. Após 44 dias, atingiu o maior número de fechamento de escolas, ou seja, $91.4 \%$ do total de estudantes matriculados com um total de 195 países que haviam fechado suas escolas, deixando cerca de 1.600.862.642 estudantes sem aulas presenciais (Figura $03-\mathrm{B}$ ).

Figura 03 - Início (A) e pico (B) do fechamento total de escolas no mundo devido a pandemia
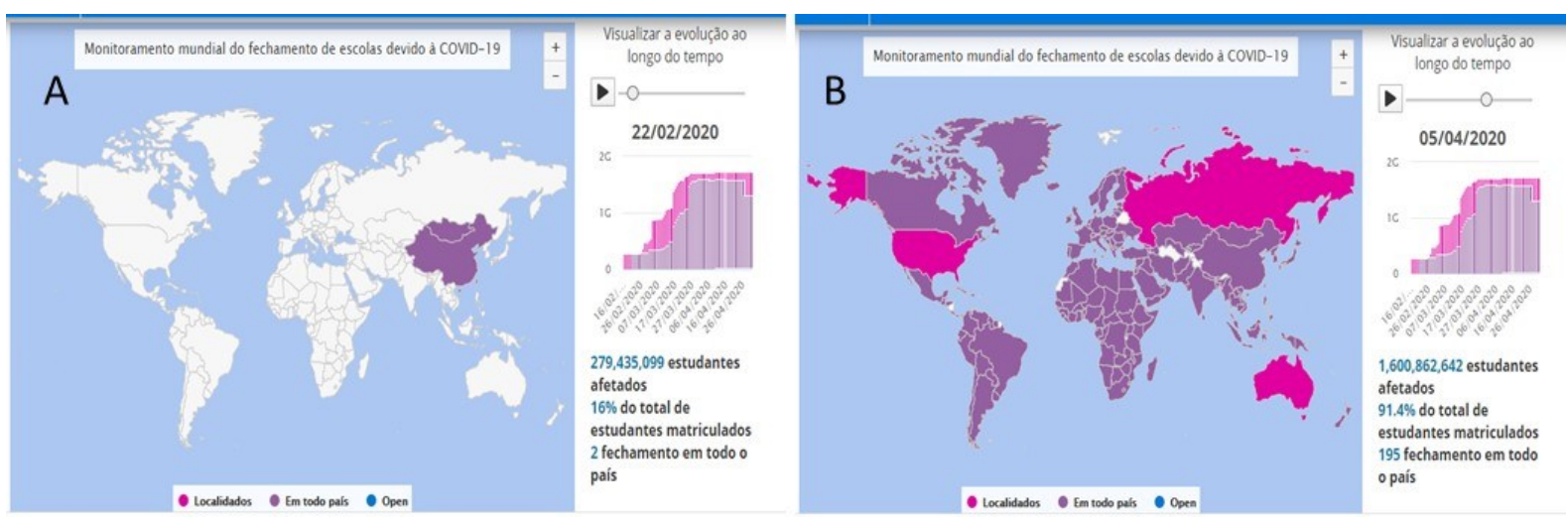

Fonte: UNESCO, 2020c

Organização: os autores.

Em ambos os países ocorreu a interrupção das atividades presenciais nas creches, escolas e universidades públicas e privadas. De forma geral, o fechamento do comércio (exceção para os serviços considerados essenciais), empresas etc., estimulou a realização do trabalho remoto para a maior parte dos trabalhadores. Com isso, a dinâmica das famílias com crianças e adolescentes tem exigido um esforço maior dos pais, responsáveis e/ou cuidadores, que também é um impacto a ser considerado.

O fechamento total das escolas e instituições de ensino no Brasil e em Cuba ocorreram oficialmente no dia 27 de março de 2020, ou seja, dois meses se passaram e os Países tiveram que apresentar propostas de enfrentamento a Covid-19 no que se refere a educação básica e ao ensino superior. No Brasil totalizaram 52.898 .349 alunos afetados, sendo $12 \%$ na educação infantil, $26 \%$ no ensino fundamental, $52 \%$ no ensino médio e $20 \%$ no ensino superior. Já em Cuba foram 2.202 .822 alunos afetados, sendo $19 \%$ na educação infantil, $28 \%$ no ensino fundamental, $38 \%$ no ensino médio e $15 \%$ no ensino superior (UNESCO, 2020c).

Dessa forma, no Brasil com a suspensão das aulas presenciais, as secretarias estaduais tomaram uma série de medidas para oferecer ensino remoto e tentar mitigar as perdas na aprendizagem dos alunos causadas pela pandemia do novo coronavírus, o que não foi diferente em Cuba com a utilização das tele aulas. 
Covid 19 e os impactos na educação: percepções sobre Brasil e Cuba
Eva Teixeira dos Santos Eros Salinas Chavez Anderson Antonio Molina da Silva Geovandir André Lordano Lucy Ribeiro Ayach Vicentina Socorro da Anunciação Ricardo Lopes Batista

Vale lembrar que em ambos os países, recaiu sobre as famílias a responsabilidade de criar as condições apropriadas a fim de que essas transmissões sejam visualizadas pelos alunos, bem como realizem as atividades orientadas, para contribuir para o cumprimento bem-sucedido dos objetivos das diferentes disciplinas.

O momento ora vivenciado nos países exigiu o planejamento ou o replanejamento a partir de informações específicas atualizadas. Portanto, foram necessárias medidas padronizadas em alguns aspectos e específicas em outros, respeitando as peculiaridades locais.

No Brasil, a partir da declaração de disseminação comunitária da Covid-19 em todos os continentes caracterizando-a como pandemia em 11 de março de 2020, a OMS - Organização Mundial de Saúde recomendou o isolamento e tratamento dos casos identificados, testes massivos e distanciamento social. Com isso, o Ministério da Saúde com a Portaria $n^{\circ}$ 188, de 3 de fevereiro de 2020, declarou Emergência em Saúde Pública de Importância Nacional, em virtude do novo Corona vírus (Covid-19). Desta forma, estados e municípios brasileiros publicaram instrumentos legais e normativos para o enfrentamento da emergência de saúde pública, incluindo a suspensão das atividades escolares.

Outra portaria (Portaria $n^{\circ} 343$ ) foi publicada pelo MEC em 17 de março de 2020, considerando a substituição das aulas presenciais por aulas em meios digitais, durante a pandemia da Covid-19, para instituição de educação superior integrante do sistema federal de ensino, sendo posteriormente alterada pelas Portarias 345 e 356/2020. Além disso, o Conselho Nacional de Educação (CNE) elucidou aos sistemas e às redes de ensino, de todos os níveis, etapas e modalidades, a necessidade de reorganizar as atividades acadêmicas por conta de ações preventivas à propagação da Covid-19. Os Conselhos Estaduais de Educação de diversos estados e Conselhos Municipais de Educação também normatizaram a orientação para as instituições sobre a reorganização do calendário escolar e uso de atividades não presenciais (BRASIL, 2020a).

A Medida Provisória do Governo Federal n 934, editada em $1^{\circ}$ de abril de 2020 (BRASIL, 2020b), estabeleceu normas excepcionais para o ano letivo da educação básica e do ensino superior decorrentes das medidas para enfrentamento da situação de emergência de saúde pública de que trata a Lei n 13.979, de 6 de fevereiro de 2020. Logo após, em 3 de abril de 2020, o MEC publicou a Portaria $n^{\circ} 376$ (BRASIL, 2020c)que dispõe sobre as aulas nos cursos de educação profissional técnica de nível médio durante a situação de pandemia da Covid-19, autorizando, em caráter excepcional, as instituições integrantes do sistema federal de ensino quanto aos cursos de educação profissional técnica de nível médio em andamento, a suspender as aulas presenciais ou substituí-las por atividades não presenciais por até 60 dias, prorrogáveis a depender de orientação do Ministério da Saúde e dos órgãos de saúde estaduais, municipais e distrital. O Ministério da Educação também realizou outras ações pontuais para a mitigação dos impactos da pandemia na educação.

É importante salientar que cada nível educacional possui parâmetros mínimos de carga horária e dias letivos, de acordo com os artigos 24 (ensino fundamental e médio), 31 (educação infantil) e 47 (ensino superior) da Lei de Diretrizes e Bases da Educação Nacional (LDB). No ensino superior, cada curso tem definida sua carga horária específica, de acordo com seu currículo e as respectivas Diretrizes Curriculares Nacionais (DCN's).

Apesar de, para conter o avanço da doença, em todos os estados do Brasil ter havido fechamento de escolas e/ou a suspensão de aulas e de no mundo, mais de 1,5 bilhão de estudantes em 165 países serem afetados pelo fechamento de escolas (UNESCO 2020b), nem todas as instituições de ensino no Brasil tomaram decisões por suspensão de aulas, houve também a adoção imediata pela continuidade de aulas não presenciais, e outras iniciaram a pandemia com a suspensão e posteriormente aderiram às aulas não presenciais, dada a perspectiva de um período longo da pandemia no país.

O cenário marcadamente controverso sobre o direcionamento de decisões quanto às aulas em todos os níveis durante a pandemia levou a muitas discussões e manifestações de dirigentes, professores, alunos e familiares sobre o que seria o ideal nesse momento de excepcionalidade.

Nessa perspectiva as instituições de ensino brasileiras passaram a utilizar como alternativas de ensino material impresso (ausência de acesso a internet) e ferramentas digitais como: Teleaula - TV aberta, 
Covid 19 e os impactos na educação: percepções sobre Brasil e Cuba
Eva Teixeira dos Santos Eros Salinas Chavez Anderson Antonio Molina da Silva

Geovandir André Lordano Lucy Ribeiro Ayach Vicentina Socorro da Anunciação Ricardo Lopes Batista

Plataformas digitais, aplicativos, Plataforma Google Classroom, outros (WhatsApp, youtube), conforme demonstrado na figura 04.

Figura 04 - Alternativas utilizadas para o ensino durante a Pandemia, por Unidades da Federação Brasil
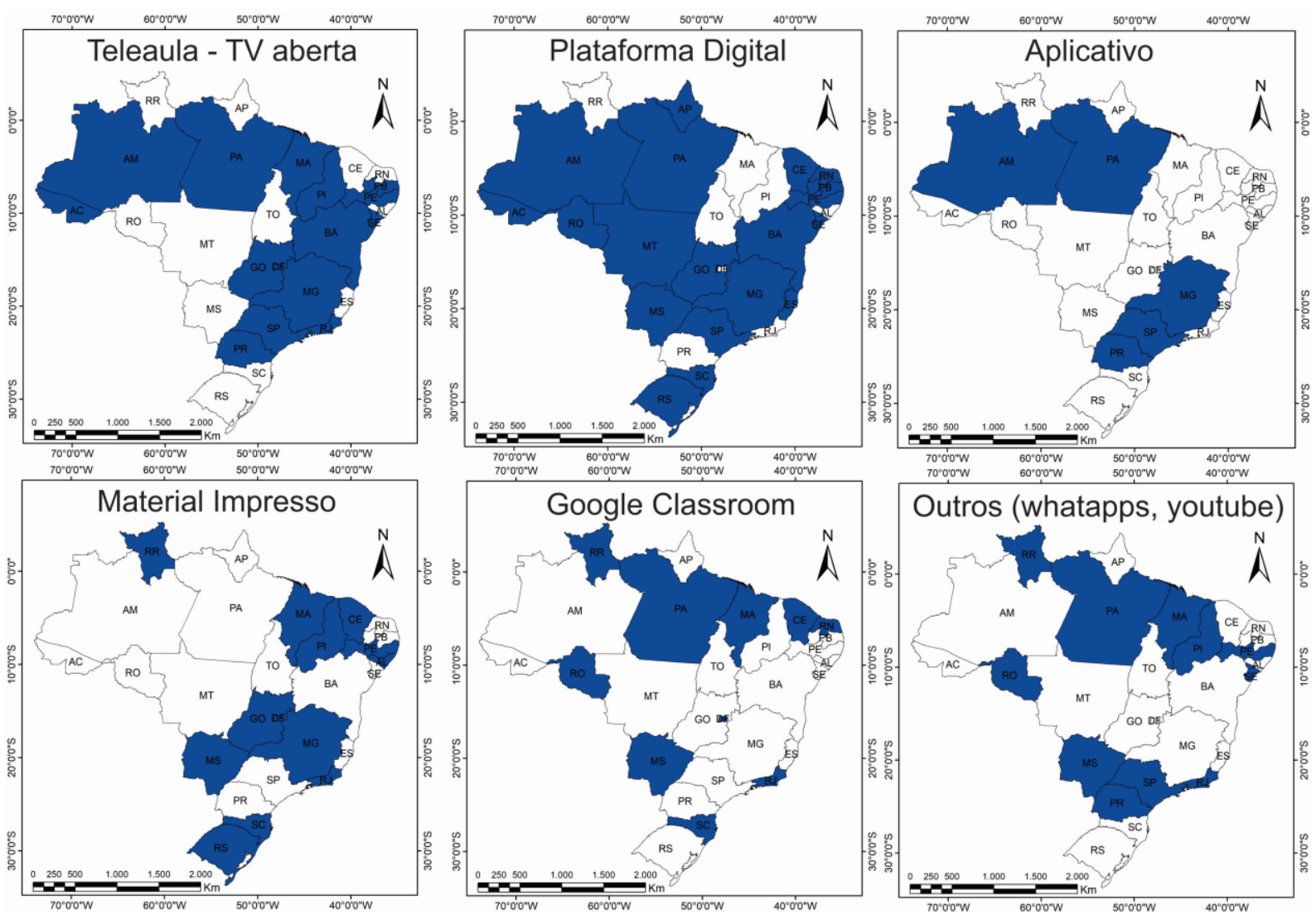

Fonte: CONSED.INFO, 2020 (atualizado em 23 de abril de 2020)

Elaboração: Os autores.

Entretanto, com a implementação de atividades a distância em todo o País, é importante considerar os dados da Pesquisa Nacional por Amostra de Domicílios Contínua - Tecnologia da Informação e Comunicação (Pnad Contínua TIC) (IBGE, 2018), divulgada em 29 de abril de 2020, que demonstra que 46 milhões de brasileiros não possuem acesso à rede mundial de computadores. No ano de 2018, no Brasil, 58\% dos domicílios não possuem computador, 33\% dos domicílios não tinham acesso algum a internet, sendo $60 \%$ entre as residências das classes D e E. $56 \%$ dos usuários de internet do Brasil acessam a rede apenas pelo telefone celular, índice que se eleva a $85 \%$ entre as classes D e E, e que perfaz $61 \%$ dentre os usuários de 10 a 15 anos, faixa etária predominante na REE (UNESCO, 2019). Apesar do número de indivíduos com acesso ter aumentado nos últimos anos, na área urbana o percentual passou de 80,2\% em 2017 para 83,8\% (Figura $05 \mathrm{~A}$ ). Na área rural passou de $41 \%$ em 2017 para $49,2 \%$ em 2018, ou seja, 50,8\% da população rural não tem acesso a internet no Brasil (Figura 05 B). 
Eva Teixeira dos Santos Eros Salinas Chavez

Covid 19 e os impactos na educação: percepções sobre Brasil e Cuba

Anderson Antonio Molina da Silva

Geovandir André Lordano Lucy Ribeiro Ayach

Vicentina Socorro da Anunciação Ricardo Lopes Batista

Figura 05 - Percentual de domicílios urbanos (A) e rurais (B) com e sem acesso a internet, segundo as grandes regiões.

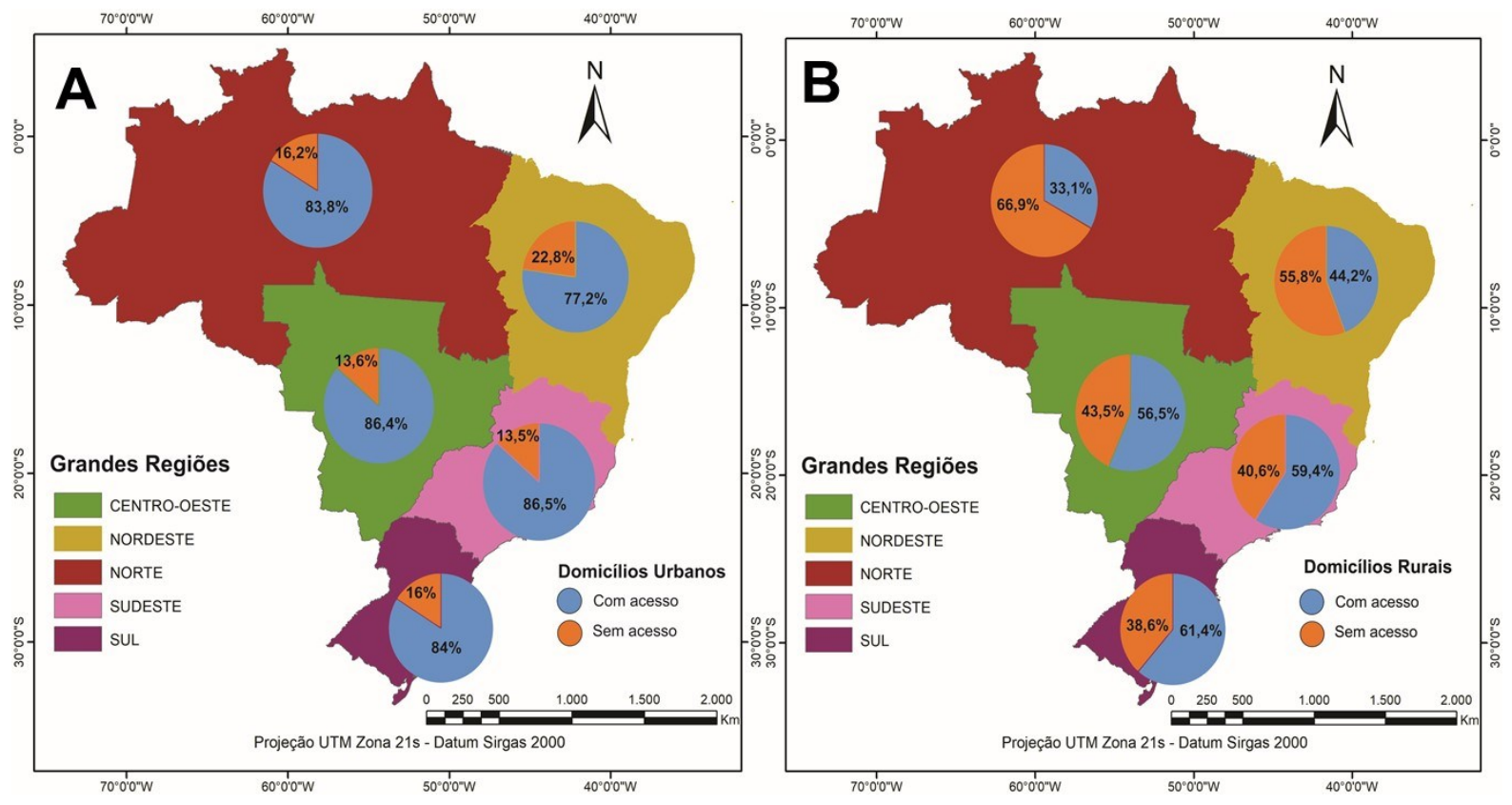

Fonte: IBGE (2018). Elaboração: os autores.

Nesse sentido, a pandemia revelou uma desigualdade social no sistema de educação, apesar de incentivar o uso das novas tecnologias na BNCC e nos currículos estaduais, há pouco suporte nas escolas públicas para que as tecnologias sejam utilizadas efetivamente. A maioria dos Estados brasileiros adotaram meios digitais para troca de materiais e informações com os estudantes (CONSED.INFO, 2020). No entanto, 46 milhões de brasileiros não possuem acesso à rede mundial de computadores, e quando observados os dados de acesso por domicílios rurais, os dados são ainda mais alarmantes, na região norte do país 66,9\% dos domicílios rurais não possuem acesso à internet, quando comparados com os dados das plataformas utilizadas, o Acre, Amazonas, Pará e Amapá adotaram plataformas digitais, porém não disponibilizaram materiais impressos aos estudantes.

Quanto a situação de Cuba, em 29 de abril de 2020, autoridades do País, dentre elas a Ministro da Educação, o Ministro do Ensino Superior, o Diretor Nacional de Ensino Médico do MINSAP e o Chefe da Diretoria de Organização e Pessoal da FAR participaram do programa de televisão e apresentaram detalhes sobre a reorganização do ano letivo em todos os níveis de ensino, os exames de ingresso no ensino superior, a convocação e dispensa do Serviço Militar e outros temas de grande interesse para a população. A Ministra da Educação ressaltou a situação do ano letivo, lembrando que em 20 de abril não foi possível reabrir as atividades de ensino, devido ao aumento de casos no País. Desta forma, os ministérios da Educação, Cultura e Instituto Nacional de Esportes, Educação Física e Recreação concordam que, uma vez reiniciado o ano letivo, que ocorrerá de acordo com o comportamento da pandemia, serão necessárias ao menos oito semanas para realizar algumas ações essenciais, divididas em etapas, sendo a primeira delas decisiva para a reunião de grupos de estudantes e professores e para a atenção e o trabalho diferenciado em cada instituição, com base nas particularidades de cada província. Após isso, as ações serão divididas e planejadas por nível de ensino, de acordo com as diretrizes curriculares do País até o retorno total das atividades.

Após a análise das informações consultadas, é possível destacar a ausência por parte dos governos dos países de um levantamento estatístico sobre a realidade, sobre as desigualdades regionais, do ponto de vista das infraestruturas nos países e sobre o acesso a internet por parte dos docentes e alunos (no caso do Brasil), uma vez que Cuba por dificuldades de acesso a rede mundial de computadores optou por tele aulas. 
Covid 19 e os impactos na educação: percepções sobre Brasil e Cuba
Eva Teixeira dos Santos Eros Salinas Chavez Anderson Antonio Molina da Silva Geovandir André Lordano Lucy Ribeiro Ayach Vicentina Socorro da Anunciação Ricardo Lopes Batista

Outro fato evidenciado é a falta de preparo dos pais para auxiliar os filhos nas tarefas escolares. De acordo com a Pesquisa Nacional por Amostra de Domicílios Contínua (PNAD, 2018), no Brasil apenas $16,5 \%$ da população possui ensino superior completo, $27 \%$ tem o ensino médio completo, a maior parte da população, $48,1 \%$ possui até o ensino fundamental. Sendo assim, sentem dificuldades e não tem o preparo pedagógico para lidar com o home homeschooling.

Além das dificuldades até aqui mencionadas, há ainda a que se refere ao trabalho do professor. As atividades não presenciais culminaram no aumento exponencial do trabalho docente, os quais possuem uma grande quantidade de alunos, com situações socioeconômicas muito distintas, que por sua vez incorre na necessidade de apresentar múltiplas estratégias de ensino como: 1. Elaboração de atividades e de conteúdos entregues nas escolas para a distribuição aos alunos sem acesso a internet; 2. Visualização de tutoriais para aprender a trabalhar com mídias digitais 3 . Elaboração de atividades e de conteúdos na plataforma online (Google Classroom); 4. Gravação de aulas disponibilizadas no Youtube ou aplicativos de conversa (WhatsApp); 5. Correção das atividades; 6 . Elaboração, aplicação e correção de provas; e 7. Acompanhamento e lançamento da frequência no diário escolar; entre tantas outras.

Sabe-se que muitas das atividades supracitadas já eram desenvolvidas pelos professores nas aulas presenciais, mas não se pode desconsiderar os novos desafios impostos em momentos de aulas não presenciais. Por exemplo, o simples acompanhamento do diário escolar pode se tornar um caos frente a dificuldade em se conferir a presença dos alunos por meio de aplicativos de conversa, além da dificuldade em preparar as aulas para plataformas digitais tão específicas.

É dado ênfase a formação da pessoa, a inteligência emocional, dosagem de razão, emoção, aprender a aprender, pensar por conta própria, tomar decisões, criatividade, raciocínio lógico, e senso crítico bem dosado, indícios que comprovam a inserção parcial da educação de ambos os países na revolução técnico científica, associado a todos os percalços da precarização do trabalho docente, infraestrutura institucional deficitária, impactos na saúde psicossocial da classe trabalhadora e demais atores sociais inseridos no processo.

A educação na perspectiva da Revolução 4.0, adverte que a obtenção e gerenciamento do conhecimento pode ocorrer fora de local, momento e horário específicos, sendo acessível e de simples localização. Os professores através do trabalho conjunto, multidisciplinar criam os conteúdos visando solucionar temáticas específicas associando inteligência emocional com conhecimento técnico. $O$ aluno, protagonista, irá impor o compasso da aprendizagem do programa de ensino executado através da inteligência artificial. As Instituições de Ensino precisam rever as estruturas curriculares, introduzir a educação Just-in-Time. Além disso, a divisão do risco financeiro será compartilhada, o sucesso ou fracasso da pessoa (funcionário) com a instituição que a formou.

De acordo com o relatório Futuro do Trabalho, do Fórum Econômico Mundial, 2018 é preciso investir em políticas educacionais capazes de acompanhar a revolução digital, uma vez que $65 \%$ de todas as crianças do planeta que entram hoje na escola terão empregos que ainda não existem. A educação será integrada e ininterrupta, configurando o professor como um agente de aprendizagem sendo que o papel das instituições de ensino deverá inserir os jovens com as habilidades requeridas pelo mercado, e no contexto de ambos os países, tais perspectivas se encontram distante da realidade materializada, sobretudo nos índices de inclusão digital, digitalização da produção, capacitação da população e muito longe de alcançar o modelo de aprendizagem 702010 que ajuda na motivação de toda a equipe, unindo o prático ao teórico, exigências da Revolução 4.0, que envolve a aprendizagem com a própria experiência, a aprendizagem com outras pessoas, a aprendizagem com cursos (UNICEF, 2018).

\section{CONSIDERAÇÕES FINAIS}

É fato que o isolamento social é necessário para a diminuição da curva do COVID-19 para não sobrecarregar o sistema de saúde, tal isolamento mudou a rotina da população de uma maneira rápida, mudando a maneira de trabalhar, fazer compras e atender as necessidades básicas, utilizando-se para isso tecnologias como, aplicativos de delivery, internet bank, WhatsApp, skype entre outros.

Diante do cenário apresentado, deve ser considerado o esforço da gestão governamental nas diferentes esferas e dos profissionais de educação para minimizar os efeitos negativos da pandemia 
Covid 19 e os impactos na educação: percepções sobre Brasil e Cuba
Eva Teixeira dos Santos Eros Salinas Chavez Anderson Antonio Molina da Silva Geovandir André Lordano Lucy Ribeiro Ayach Vicentina Socorro da Anunciação Ricardo Lopes Batista

no setor educacional. Entretanto, as medidas até agora adotadas pelo governo brasileiro denotam um otimismo exagerado quanto aos resultados da adoção da educação à distância, notadamente quanto aos aspectos qualitativos que não estão sendo considerados. Além disso, outro fato precisa ser considerado: a defesa da educação a distância na educação básica que vem se apresentando durante a pandemia.

Em Cuba, considerando a adoção de tele aulas pelos canais estatais, atrelado ao melhor nível de escolaridade dos responsáveis pelos alunos, a situação aparenta ter impactos um pouco menores que no Brasil. Outro fato é que o ano letivo de Cuba ocorre de setembro a julho e dessa forma já tinham a maior parte das atividades realizadas, facilitando o término, via canais de televisão. Nestes canais são veiculadas campanhas a população sobre a importância do acompanhamento aos estudantes durante o período de suspensão das aulas.

O tempo decorrido diante da situação de pandemia ainda não permite afirmar com exatidão quais são as melhores decisões em prol da educação nesse momento, uma vez que a paralisação total das aulas, juntamente com o isolamento pode desencadear outros problemas de ordem social e psicológicas. Porém, cabe apontar, diante dos dados, as evidências do impacto negativo no processo ensinoaprendizagem, quanto à dificuldade de acesso às tecnologias, que induzem à exclusão, a necessidade de preparação e planejamento pedagógico por parte dos professores, além de ratificar a dificuldade dos professores na promoção do auto estudo e construção do conhecimento pelos alunos, bem como a falta de escolaridade dos responsáveis para o acompanhamento dos alunos em atividades a distância.

Assim, cabe indagar sobre quais as potenciais lições decorrem da pandemia da Covid-19 para educação brasileira e cubana. A realidade não pode ser explicada por um conceito universal, pois cada país, cada cultura e cada pessoa, são únicos e vivem uma experiência diferenciadas, sobretudo no contexto educacional brasileiro, país de dimensão continental e disparidades regionais.

Por fim, vale ressaltar que o processo de isolamento social é sempre discriminatório. Na conjuntura educacional, observa-se que tem sido mais difícil para alguns grupos sociais do que para outros e impossível de conceber para um grande grupo de atores sociais, com necessidades básicas que já precediam a pandemia, sendo ainda mais agravada com ela, como é o caso da população em vulnerabilidade social.

\section{REFERÊNCIAS}

BRASIL. PORTARIA N 343, DE 17 DE MARÇO DE 2020. Dispõe sobre a substituição das aulas presenciais por aulas em meios digitais enquanto durar a situação de pandemia do Novo Coronavírus - COVID-19. 2020a. Disponível em: http://www.in.gov.br/en/web/dou/-/portaria-n-343-de-17-de-marcode-2020-248564376. Acesso em 03 mai.2020.

BRASIL. MEDIDA PROVISÓRIA N 934, DE $1^{\circ}$ DE ABRIL DE 2020. Estabelece normas excepcionais sobre o ano letivo da educação básica e do ensino superior decorrentes das medidas para enfrentamento da situação de emergência de saúde pública de que trata a Lei $n^{\circ} 13.979$, de 6 de fevereiro de 2020. DIÁRIO OFICIAL DA UNIÃO. 01/04/2020, Edição: 63-A, Seção: 1 - Extra, pg. 1. 2020b.

CONSED.INFO. Página de acompanhamento das redes estaduais de educação durante a pandemia do novo coronavírus. ENSINO REMOTO. Disponível em: https://consed.info/ensinoremoto/. Acesso em 06 mai. 2020.

CUBADEBATE. COVID-19 en Cuba: Definiciones del presente curso escolar. Disponível em: http://www.cubadebate.cu/noticias/2020/04/29/covid-19-en-cuba-definiciones-del-presente-cursoescolar-video/\#.XrReBKhKjIV. Acesso em 03 mai. 2020.

FARIAS, Heitor Soares de. O avanço da Covid-19 e o isolamento social como estratégia para redução da vulnerabilidade. Espaço e Economia [Online], $17 \mid 2020$. Disponível em: http://journals.openedition.org/espacoeconomia/11357. Acesso em: $30 \quad$ abri. 2020. https://doi.org/10.4000/espacoeconomia.11357 
Covid 19 e os impactos na educação: percepções sobre Brasil e Cuba
Eva Teixeira dos Santos Eros Salinas Chavez Anderson Antonio Molina da Silva

Geovandir André Lordano Lucy Ribeiro Ayach Vicentina Socorro da Anunciação Ricardo Lopes Batista

ORNELL, Felipe; SCHUCH, Jaqueline Bohrer; SORDI, Anne Orgler; KESSLER, Felix Henrique Paim. Pandemia de medo e CoVid-19: impacto na saúde mental e possíveis estratégias. Revista Debates In Psychiatry - Ahead of print. Ano 10. 2020, p. 1-7. Disponivel em: https://d494f813-3c95-463a-898cea1519530871.filesusr.com/ugd/c37608_8b90d53e74b548239783585d78320f21.pdf Acesso em: 01 mai. 2020.

PNAD, IBGE. Acesso a internet e à Televisão e Posse de Telefone Móvel Celular para Uso Pessoal 2018. Rio de Janeiro: IBGE (Instituto Brasileiro de Geografia e Estatística), 2020. Disponível em: https://www.ibge.gov.br/estatisticas/sociais/populacao/17270-pnadcontinua.html?edicao=27138\&t=resultados Acesso em 05 mai. 2020.

UNESCO. Centro Regional de Estudos para o Desenvolvimento da Sociedade da Informação. Pesquisa TIC Domicílios. São Paulo. 2019. Disponível em https://www.cetic.br/media/analises/tic domicilios 2018 coletiva de imprensa.pdf Acesso em 01 mai. 2020.

UNESCO - Organização das Nações Unidas para a Educação, a Ciência e a Cultura. Coronavírus: UNESCO e UNICEF trabalham para acelerar soluções de aprendizagem a distância. 2020a. Disponível em: https://nacoesunidas.org/coronavirus-unesco-e-unicef-trabalham-para-acelerar-solucoes-deaprendizagem-a-distancial. Acesso em 17 abr. 2020.

UNESCO. United Nations Educational, Scientific and Cultural Organization. Schools are more than just a platform for knowledge, UNESCO webinar highlights. 2020b. Disponível em: https://en.unesco.org/news/schools-are-more-just-platform-knowledge-unesco-webinar-highlights. Acesso em 01 Mai. 2020.

UNESCO. United Nations Educational, Scientific and Cultural Organization. Covid-19 educational disruption and response. COVID-19 Impact on Education. 2020c. Disponível em: https://en.unesco.org/covid19/educationresponse Acesso em 01 Mai. 2020.

UNICEF. Com evolução tecnológica, $65 \%$ das crianças terão empregos que ainda não existem, diz CEPAL. 2018. Disponível em: https://nacoesunidas.org/com-evolucao-tecnologica-65-das-criancasterao-empregos-que-ainda-nao-existem-diz-cepal/. Acesso em: 03 mai. 2020 